\title{
The Development and Current Situation of the Early Intervention for Children with Developmental Delay in Taiwan
}

\author{
Pi-Hsia Huang \\ Children's Bureau of the Interior \\ Taiwan
}

\begin{abstract}
In this thesis, it introduces the development and current situation of the early intervention for children with developmental delay in Taiwan. First, it gives an explanation on the policy evolution on this issue after the 1993 amendment of "Child Welfare Law" of ROC, and then it depicts the four steps in the process of early intervention for children with developmental delay, i.e. "Screening and Detection", "Report and Referral", "Team Evaluation", "Intervention Service". Thereafter, a detailed explanation on the major models of intervention service and a plan on the direction of future development are established.
\end{abstract}

Key Words : children with developmental delay, early intervention service

The early intervention service(EIS) for children with developmental delay is a booming welfare issue in Taiwan in recent ten years and is also one of the most rapidly growing social policies. Some civil organizations are starting to be engaged in the promotion of early intervention since early 1990s. Since the Child Welfare Law was amended in 1993, the EIS for children with developmental delay has been officially taken under the protection of law and the government in Taiwan is also planning to promote this service. Furthermore, the EIS is an integrated and inter-discipline service which puts an emphasis on the children's right to develop and thus requires the integration of medical, educational and social welfare departments to provide comprehensive professional service. The labor division of this service in the government involves 3 government departments, i.e.

Pi-Hsia Huang, Director of Children's Bureau of the Interior Li-Ming Rd., Sec 2, Taichung, Taiwan, 408, R.O.C.

cbi888@cbi.gov.tw
Department of Health, Ministry of Education and Child Welfare Bureau Ministry of the Interior (CWB).

\section{History and Development}

In earlier years, the society in Taiwan did not attach too much importance to the development of children, especially for those who commonly hold the traditional opinion about a late boomer that "greater talent shows itself late in life" and that "they would have an enlightenment one day" which resulted in that many children with developmental delay missed the opportunity to receive appropriate education and treatment at the earliest possible time. Due to the great efforts exerted by various civil social welfare organizations in the early 1990s, the development of EIS for children with developmental delay and their family has been taken under the protection of the 1993 amendment of Child Welfare Law (Wang, 1996). The 
Ministry of the Interior has also established a "Work Team for the Promotion of the Development of early intervention for children with developmental delay", which adopts an interdepartmental approach to coordinate the efforts of medical, educational and social welfare departments to promote the development of early intervention. In 1997, "the Plan for the Development of Early Intervention Service for Children with Developmental Delay" was formulated in which the procedure of EIS was divided into four steps: "Screening and Detection", "Report and Referral", "Team evaluation", "Intervention Service". Presently, the contents and procedures of EIS in Taiwan are based on this plan.

In 1999, CWB was established as the central competent authority and issues concerning the development of the EIS for children with developmental delay which were originally administered by the Department of Social Affairs, Ministry of the Interior had been put under the administration of this bureau. And the Child Welfare Law and Youth Welfare Law were amended and merged as Child and Youth Welfare Law in 2003 to go along with the international trend. According to article 6 of the detailed rules for the implementation of this law, "children with developmental delay" is defined as children with suspected abnormalities or expected to develop suspected abnormalities on various aspects such as cognition development, physiological development, language and communication development, psychological \& social development or self-care skills and have undergone the evaluation process in any hospital approved by the competent authority in charge of healthcare issues and have been confirmed with such abnormalities and issued a certificate. Furthermore, according to this very same article, "early intervention service" is defined as any necessary healthcare and treatment, education, consultation, referral, accommodation and other services that are provided by social welfare, healthcare, education professionals in team work according to the individual needs of children with developmental delay under the age of 6 and their families.
The official implementation of EIS by Ministry of the Interior can be dated back to 1995, and this service has been implemented for more than a decade. During this period, apart from the enforcement of the relative regulations in the Child and Youth Welfare Law, CWB has also formulated several supportive plans in response to the requirements of the service practice, such as: "AtHome Service for Children with Developmental Delay", "Plan of Professional Guidance Tours for Nursery Institutions to Accept Children with Developmental Delay", "the Subsidy of Intervention Expenses to Children Developmental Delay" etc. Presently 25 counties/cities of Taiwan have established their "Early Intervention Reporting Referral Center for Children with Developmental Delay", and Department of Health has also established "Center of Team Evaluation for Children's Development" in various counties/cities throughout Taiwan. Furthermore, many counties/cities have intervention service organizations. With the establishment of these relative units which cover the fields of social science, healthcare and education, a service delivery process of the whole early intervention has been formed to provide relative services to children with developmental delay and their families.

\section{Connotation}

The early intervention is a human-based, complete and active caring measure that adopts integrated and multi-discipline services to solve various medical, educational, family and social problems relating to children with developmental delay or developmental disabilities. On one hand, early intervention solves these related problems; on the other hand, it can exploit the latent energy of the children and reduce the severity of disabilities in the future and effectively cut the long-term social cost (Guo, 1996). In the "Plan of Early Intervention Service for Children with Developmental Delay" promulgated by Ministry of the Interior, "Screening and detection", "Report and Referral", 
Table 1

Chronicles of the Development of Early Intervention Service for Children with Developmental Delay

\begin{tabular}{cl}
\hline Time & \multicolumn{1}{c}{ Events } \\
\hline 1993 & $\begin{array}{l}\text { The amendment of Child Welfare Law was passed, the EIS for children with developmental delay and their family was taken under the } \\
\text { protection of this law for the first time. }\end{array}$ \\
\hline 1994 & $\begin{array}{l}\text { The byelaw for the implementation of Child Welfare Law was amended, and the definition of "developmental delay" and "early } \\
\text { intervention" were defined. }\end{array}$ \\
\hline
\end{tabular}

- The Ministry of the Interior established a work team for the promotion of the development of early intervention for children with mentally developmental delay, which adopted an interdepartmental approach to promote the development of early intervention.

1995 - The national healthcare insurance was established.

- The Department of Health opened the "Children Preventive Healthcare Service."

The Department of Health and the Ministry of Education jointly opened the "Screening plan for amblyopia in preschool children."

1996 The Department of Health, together with education and social welfare system jointly opened "Health Management Plan for Children in Kindergartens and Nurseries."

- The amendment of the Special Education Law was passed and the school age of special education students was adjusted downward to 3. Such change will be gradually fulfilled in 6 years.

1997 The Ministry of the Interior selected Taipei City, Taichung County, Tainan City, Kaoshiong City and Hualian County to carry out trials on "service for the report \& referral of early intervention to children with developmental delay and the case management."

- The Department of Health selected Taipei municipal maternal and child hospital, the affiliated hospital of Tainan Cheng Kung University, the affiliated hospital of Kaoshiong Medical University, the affiliated hospital of Hualian Tzu-Chi Medical University

1997 to set up the "Center of Team Evaluation for Children's Development for Children with Developmental Delay."

- The Ministry of the Interior promulgated "the Implementation plan of early intervention service for children with developmental delay."

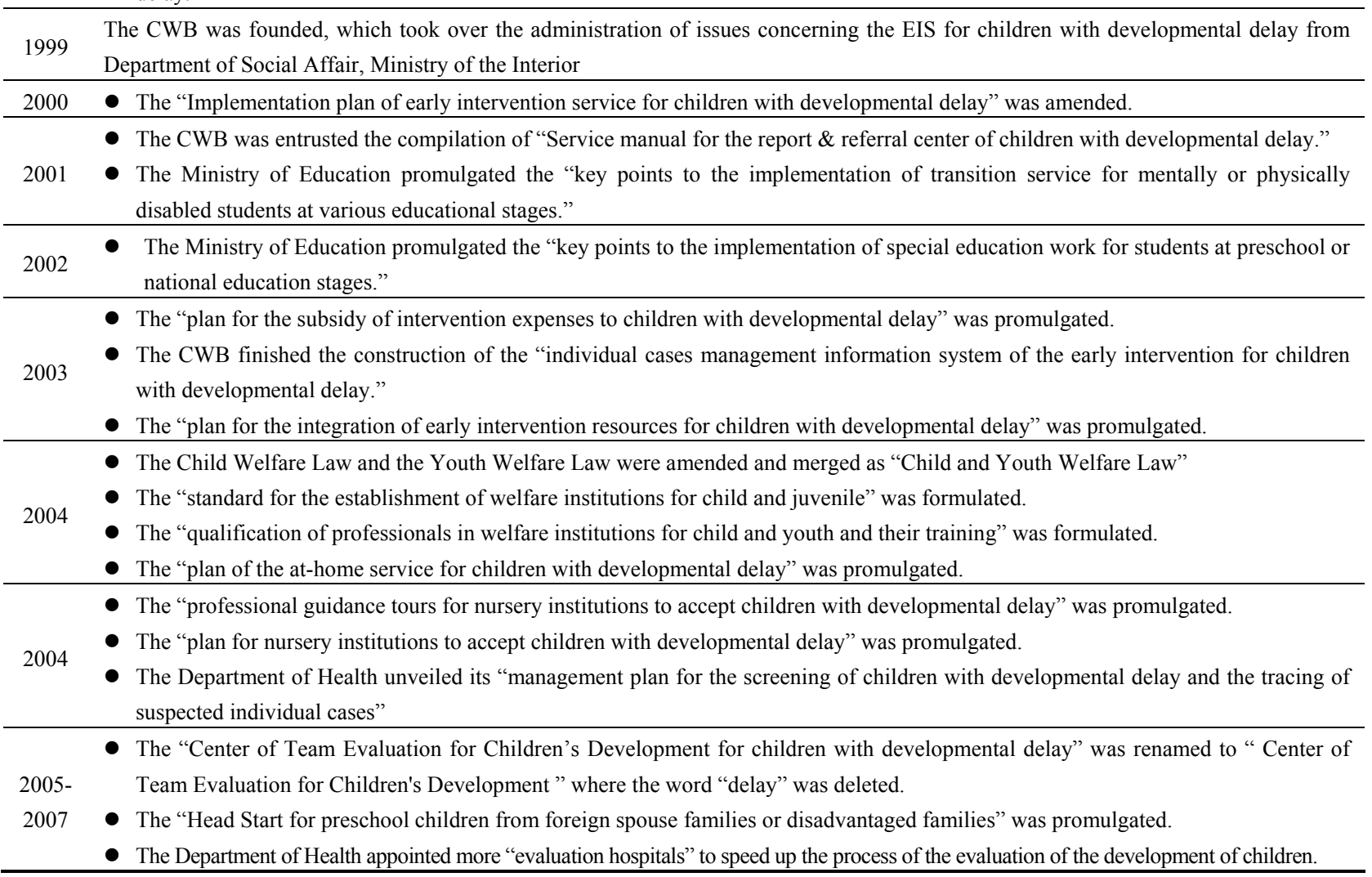

Note. From Child Welfare Data [public notice] Child Welfare Bureau, 2006 
"Team evaluation" and "Intervention Service" are defined as the main connotation of this service.

However, from the viewpoint of tertiary prevention, more preventive measures should be done before children with developmental delay are discovered; that is, to prevent the developmental delay from happening. We will clarify this viewpoint by "Prevention", "Screening and detection", "Report and Referral", "Team evaluation" and "Intervention Service" respectively.

\section{Prevention}

The focus of work at this stage should be placed on the promotion of health and special protection. We should strengthen the propaganda of relevant knowledge in the general public, including the execution of premarital health examination, eugenic measures, etc. As regards to possible causes that might lead to the developmental delay of children, we should strengthen the preventive measures from the beginning. The preventive services available to mothers at prenatal, perinatal and postnatal periods are as follows: 1. Prenatal: apart from strengthening the propaganda of relative knowledge such as instructing the public to avoid consanguineous marriage and elderly primiparity or primiparity of over young age to take premarital health examination, to seek genetic consultation, we also need to instruct the public to prevent premature labor and various infections, to quit smoking, to abstain from alcohol and drug abuse, to take antenatal examination, etc. Furthermore, we should strengthen the prenatal care and reduce the occurrence of high-risk neonate. Presently, the national healthcare insurance provides 10 free antenatal examinations, and the examination items include: anemia, thalassemia, platelet count, treponemal tests for syphilis, $\mathrm{Rh}$ factor test, ultrasound screening and ABO blood type test, serum test for hepatitis $B$, rubella antibody test. 2 Perinatal: the emphasis should be placed on the prevention of birth injury and emergency treatment of various emergent situations. Perfect birth care can be helpful to reduce the occurrence of developmental delay in children. 3. Postnatal: importance should be attached to the nutrition and disease prevention of the neonate after birth. The baby should be inoculated and be provided with a good environment and cultural stimuli and familial affection.

\section{Detection and Screening}

The critical point for early intervention lies in the detection and intervention at the earliest possible time. Anyone who has the opportunity to contact children, including parents, babysitters, medical professionals, educational professionals and social workers, should take their responsibility to observe whether they have any mental or physical abnormalities at any time

By means of antenatal examination, screening tests for congenital metabolism disorders in neonate, monitoring of high-risk babies, periodical physical health checks on children, tracing of the development of neonate, screening activities through baby games, parental education provided by the community and the implementation of public hygiene education, whether a child is with any development problems can be discovered clinically in our country. At its foundation in 1995, the national health insurance provided children between the ages of $0-4$ with 6 times of preventive healthcare service (the evaluation of the development of children is one of the examination items of this preventive healthcare service); from July of 2004, the target group of this service has been extended to children under the age of 7 , and the times of service had been increased to 9. According to data of 2004, with the help of the inoculation schedule, the utilization rate of this service in children under 3 reached $72.45 \%$. In order to realize the detection of children with developmental delay before they attain the age of 3 , the Department of Health put forward the "management plan for the screening of children with developmental delay and the tracing of suspected individual cases" which adopted the "check list for preschool children in Taipei," a check list developed by ZHENG, a psychologist in Taipei Veterans General Hospital, as a tool to carry out initiatory 
screening. The public health nurses of each health station in every village, town or city had been requested to strengthen the screening work when they carry out inoculations or visit families. And the "check list for preschool children in Taipei" has become one of the initiatory bases commonly used by the primary level workers.

The utilization rate of this health examination in children of ages 3-6 decreased because these children didn't have to receive inoculations. As most children of this age level have been sent to kindergartens or nurseries, the main sites of screening work will be shifted to kindergartens and nurseries. Therefore, the Ministry of Education and CWB have actively planned and implemented on-the-job training for preschool education teachers, child care workers and babysitters so as to make them become more sensitive to the development of children and can screen out those children with developmental delay. Upon their entrance into kindergartens or nurseries, children will be requested to present their physical and mental health cards in cooperation with healthcare units and to receive overall examination at least once per two years. And the kindergartens (nurseries) will offer health management materials for the children. The kindergartens will suggest a timely referral for children to seek medical advice if any abnormality is found.

\section{Report and Referral}

"Report and Referral" is the pivot of the whole service delivery process of early intervention. Since 2001, more than 10 thousands suspected cases of children with developmental delay have been reported and required to undergo examination. The case report of children with developmental delay adopts a responsibility system, when a suspected case of developmental delay is discovered, the parents or relevant people shall report to Early Intervention Reporting Referral Center according to Article 22 of the Child and Juvenile Welfare Law, "Various welfare, educational or medical institutions shall report to the competent municipality, county(city) authorities upon their discovery of suspected case of child with developmental delay or child/youth with physical or mental disabilities; and the competent municipality, county(city) authorities shall establish a dossier for the received materials. Whenever necessary, the services on transfer to appropriate organizations will be provided".

When a child with developmental delay that needs assistance is discovered, apart from reporting to the competent authorities, a dossier shall be established for relevant materials. The transfer of the child to appropriate service units, which will facilitate the prompt assistance to the individual case, is even more important. Presently, the CWB has guided 25 local governments in the establishment of Early Intervention Reporting Referral Center for Children with Developmental Delay and in the establishment of a single window to handle affairs concerning children with developmental delay in each county (city). The Report and Referral centers will plan, collect and sort materials concerning children with developmental delay and handle the following services, so as to facilitate the referral works. (a) take cognizance of the reports of individual cases. (b) give assistance to the evaluation and transfer of each individual cases. (c) assign for intervention services. (d) periodically tracing and management of each individual cases. (e) transition service for school attendance. (f) the relocation or settlement of each individual cases. (g)the input operation of materials, evaluation report, the status of relocation and service; dossier management. (h) other services concerning the consultation of relocation, referral, tracing and guidance.

Apart from the above-mentioned work focuses, Lin (2001) also integrated services of the Early Intervention Reporting Referral Center for Children with Developmental Delay of counties (cities) in Taiwan and found they can be divided as ordinary services and supplementary services. The ordinary services include: taking cognizance of report, consultation to individual cases, propaganda and resource and referral; the supplementary services include: evaluation assistance, 
parental education, growth group, study and training, expenses subsidies, transition services, itinerant guidance, home visit, initial screening of individual cases, placement tracing, at-home service, the construction of reporting net-work, web pages and database system, administration coordination, special education service, case management, day care, research, professional team evaluation, family consultation, etc.

The Early Intervention Reporting Referral Center for Children with Developmental Delay was established on the basis of public model or outsourcing model. The public model is defined as a model in which the Bureau of Social Affairs handles reports, other issues concerning the report and referral will be contracted to civil organizations. While in the outsourcing model, the report and referral and the case management will all be contracted to civil organizations pursuant to the Government Procurement Law. Both of the business handling has its own advantages and shortcomings, and the biggest difference lies in that if the report and referral works are executed by a government department, they will be facilitated by the compulsory public power. As regards to report and referral works executed by state-owned and private-run organizations or in the form of outsourcing services, the management handling of subsequent tracing of each individual cases and the follow-up intervention services will be easier.

\section{Team Evaluation}

The establishment of Center of Team Evaluation for Children's Development is one of the characteristics of Taiwan in its promotion of early intervention for children with developmental delay. If a child with the phenomena of suspected developmental delay is founded during initial screening, he will be sent to the Center of Team Evaluation for Children's Development or an evaluation hospital to receive a complete symptomatic and functional evaluation. In 1997, the Department of Health set up "Center of Team Evaluation for children with developmental delay" in selected regional large hospitals of Taipei, Taichung, Tainan, Kaoshiong and
Taitung to promote the relating medical services for children with developmental delay. In July of 2005, the “Center of Team Evaluation for Children's Development for children with developmental delay" was renamed to "Center of Team Evaluation for Children's Development", in which the word "delay" was deleted to prevent the children from being labeled too early and to reduce the complaints from the parents. On the other hand, for the convenience of parents to bring their child to see a doctor and to reduce their waiting time, to cooperate with the patients transfer system of national health insurance, besides the existing 29 "Center of Team Evaluation for Children's Development", additional 72 evaluation hospitals will be appointed in 2007 to speed up the evaluation of the development of children.

The processes of the evaluation of children development and the operation models of professional teams of various hospitals are not completely the same. Presently, the way in which the domestic "Center of Team Evaluation for Children with developmental delay" is carried out, including the diagnostic approaches of joint outpatient clinics and special outpatient clinics and it adopts the approach of the consultation of professional medical teams. Departments involved in the evaluation and diagnosis include department of child psychiatry, department of child neurology, department of otolaryngology and department of rehabilitation. Physical therapists, occupational therapists, speech therapists, social workers, clinical psychologists and special education teachers also get involved in the evaluation process. Different departments or therapists are selected for the evaluation according to the individual development degree of each child. The diagnosis of a special outpatient clinic will mainly depend on diagnosis of any department among department of child psychiatry, department of child neurology, department of pediatric and department of child rehabilitation. The special outpatient clinics will open each week at specific consulting hours and specifically provide evaluation service to children with developmental delay; and if necessary, these children will be referred to other department for further diagnosis or evaluation. 
Presently, evaluation measure commonly used in hospitals in Taiwan include BSID-II (The Bayley scales of Infant Development II), WPPSI (Wechsler Preschool and Primary Scale of Intelligence),CDIIT (Comprehensive Developmental Inventory for Infants and Toddlers), DDST (Denver Developmental Screening Test), CCDI (Chinese Child Development Inventory), etc, as the evaluation includes medical assessment, genetic screening, neurological measurement, psychology development, motor development, behavior assessment, ability of social adaptation and family assessment.

Both joint outpatient clinics and special outpatient clinics have their own advantages. For example, as for the team evaluation in joint outpatient clinics, every suspected case of child with developmental delay can seek consultation from 3 doctors. The advantage of this approach is that parents can get a relatively comprehensive evaluation without traveling between hospitals and home. The advantages of special outpatient clinics is that they are available everywhere, and that might reduce the waiting time to receive evaluation. To the hospitals with insufficient resources, this can be an appropriate approach.

\section{Intervention Services}

After a child with developmental delay has undergone the evaluation and diagnosis procedures of a medical unit and developmental delay has been determined, social workers of various Early Intervention Reporting Referral Center will recommend appropriate intervention unit according to the comprehensive evaluation report issued by the hospital and the needs of the child and his/her family. At this stage, the manager of the individual case will invite experts and parents to take part in the intervention meeting to draft the Individualized Family Service Plans (IFSP) together. Children with developmental delay and their families tend to have multiple needs due to the developmental delay of the children. For example, they might need to arrange for medical rehabilitation, apply for school admission, attend after-school child care, apply for economic allowance, attend parental education programs, etc; all of this must be arranged concurrently. Without the assistance of proper resources, these needs will give rise to even more complicated problems. The manager of the individual case is especially important for his/her role in the integration of resources and his/her role as a connector. Wan (1997pointed out that the early intervention is to provide inter-discipline services by teamwork. The referral process is indispensable to connect services and the needs of individual cases. To avoid interruption and discontinuity of services or the redundant use of resources, and to maintain the cooperative relationships among various departments, a whole set of complete materials and a service plan must be established for each individual case while the referral is in process so as to know the individual case better and to achieve the desired service effects. The formal and informal systematic arrangements of resources according to the needs of each individual case will indirectly benefit members of the family or the primary group due to this integrated systematic service.

\section{The Current Situation of the Implementation of the Early Intervention Service}

The intervention service is the last step in the process; however, it is the most important step. The services includes: the provision of medical rehabilitation, education services and family services; the service providers include hospitals, kindergartens, nurseries, early intervention organizations. Therefore, we will explain what the EIS in Taiwan are from the following 3 major aspects respectively: medical sites, preschool education and early intervention organizations, and then give a concise account of the achievement of the EIS in Taiwan.

\section{Medical Sites}

The major works of medical places are handling issues concerning the early intervention evaluation and 
medical rehabilitation services, which include medical services such as: evaluation and identification; ordinary or special treatment, physical, occupational and speech therapies; sensory integration, nutrition guidance, psychological and behavioral therapies, etc. As regards to the major models of EIS in medical places, there are individual therapy, group therapy, day care, etc, among which the cost individual therapy would usually absorbed by the national healthcare insurance and parents won't be charged any extra expenses. These will be described respectively as follows:

Individual therapy: based on the main problems or disabilities of the child, professional therapist will provide part-time therapeutic programs to him/her, each lesson will last approximately 30-40 minutes.

Group therapy: This also consists of part-time programs, usually therapeutic activities concurrently participated by several children with similar disability conditions under a therapist, or therapeutic activities designed and organized by the cooperation of 2 therapists of different specialties, for example, an occupational therapist and a speech therapist. Furthermore, some hospitals might open group programs for children with special needs, such as: Highfunctioning autism (HFA) group, hyperkinetic children group, etc.

Day care: The children will receive treatment in a hospital for half day (usually in the morning) each day, 4-5 days each week. The classes are closely scheduled; usually the parents are requested to accompany their children to participate in the programs. Despite that classes are scheduled by members of the team together, most people in charge are special-education teachers, clinical psychologists or occupational therapists (Yi, 2004.)

\section{Preschool Education and Early Intervention Organizations}

Presently, major places for children with developmental delay between 0-6 include kindergartens, nurseries and social welfare organizations, and their main contents of service include services such as: educational placement, cognitive training, movement, communication and expression, social abilities, games and individualized education programs(IEP) designed for children with developmental delay, and the providing of study approaches for inclusive education, transition services, parent education, etc. Currently, the education resources of early intervention in Taiwan region are available in the following forms:

Preschool special education class: Preschool special education classes opened by affiliated kindergartens of special education schools or primary schools will recruit children with developmental delay or children with mental or physical disabilities between 3-6, and the enrollment of such children should undergo the evaluation of a committee responsible for education and placement of special students under the bureau of education of local governments.

Day care classes under intervention organizations: Children attending these classes will receive education for a full day or half day in an early intervention organization or an organization for those mental or physical disabled under the instruction of special care workers or special education teachers in the form of group education. Usually the ratio of teacher to students is 1:3-4, and the age of these children mainly ranges from 2 to 6 .

Time series intervention: Preschool children or children not receiving education placement who attend this intervention will take 1-3 intervention classes each week according to their individual needs, each class will last approximately 1 hour. Usually this intervention will be provided by early intervention organizations or organizations for mental or physical disabled in the forms of individual courses or group courses. Presently, there are approximately 112 intervention bases throughout Taiwan which provide various intervention services which meet the needs of children with developmental delay by professionals such as physical therapists, occupational therapists, speech therapists, clinical psychologists, etc.

Inclusion education in kindergartens or nurseries: This kind of education mainly recruit ordinary children 
while at the same time also recruit children with developmental delay and place them in an ordinary class to carry out education. Usually these classes are comprised of relatively more students with a teacherstudent ratio 1:10-15 and in such a class children with special needs will have the opportunity to interact with ordinary children. This form of placement is one of those actively promoted by the government in recent years.

\section{Service Plans Relating to Children with Developmental Delay by the CWB}

Neither intervention approaches of the medical model nor that of the center model can provide a full coverage of services that meet the needs of children with developmental delay and their families. Therefore, the CWB has been dedicated to the planning of childrenoriented policies which fulfill the needs of their families in the hope that such policies will support and promote the development of the children. The following is the explanation of relevant supporting measures presently available concerning the early intervention of children with developmental delay introduced by the CWB.

The plan of the at-home-service for children with developmental delay: For those children that can not attend kindergartens, nurseries or early intervention organizations to receive EIS due to various factors such as the deficient ability of their guardians, the inconvenience of transportation, lack of education resources, economic difficulties, etc. The early intervention professionals and organizations will form teams to provide at-at-home services to them. Available service items are as follows: (a) Provide home intervention evaluation and training on cognition, language, sociality, self-care ability in life, etc by professional teams. (b) Provide instructions on parent education and strategies on the promotion of the development ability of children. (c) Provide various intervention trainings, links to aid resources and medical resources according to evaluation suggestions. (d) Provide intervention services according to evaluation and suggestions on the child's needs and living environments and community resources. (e) Give instructions to major guardians on trainings of simple and easy movements and the operation and use of living aids.(f) Improve the major guardians' knowledge and skills of education and nursing. (g) Provide emotional support, service consultation, referral or transition of resources to family members. (h) Other necessary services based on the evaluation results.

Implementation plan for the subsidy of intervention expenses to children with developmental delay: In order to lighten the economic burden of families with children with developmental delay and assist them to maintain their family function, parents with children attending governmental or private early intervention institutions, welfare organizations for mental or physical disabled, appointed medical units engaging in early intervention with approval from the department of health or local governments or other units or organizations approved by local governments to receive early intervention are entitled to the application of subsidies for intervention training expenses and transportation expenses. If the subsidy receiver belongs to low-income families, he/she will be entitled to a maximum subsidy of NT\$ 5000 per month; if not, he/she can get a maximum subsidy of NT\$ 3000 per month.

The plan for professional guidance for nursery institutions to accept children with developmental delay: It is the best choice for children with developmental delay to attend ordinary classes with ordinary children together; however, "normal" teachers or nursing workers often lack the experiences of instructing special children. Therefore, the CWB had promoted an itinerant guidance for nursery institutions to accept children with developmental delay with the help of professional teams, so as to improve knowledge and skills of the teachers and nursing workers and to assist kindergartens and nurseries in adopting diversified and elastic approaches and thus to build special education into the education and courses of ordinary classes. This way, children with developmental delay can get the best education services. The professional guidance tours of professional teams gives periodical visits to kindergartens and nurseries by 
experienced special education teachers, nursing workers, other professionals with relating certificates to give instructions and guidance to these kindergartens and nurseries. The available service items include: (a) To make up IEP and put them into practice. (b) To give strategies and suggestions on the promotion of the development ability of the children. (c) To assist teachers in seeking resources and referral service. (d) To improve relevant knowledge and skills. (e) To assist organizations with activities and lectures on child education and parental education. (f) To publicize the notion of inclusion education, assist teachers in training children with developmental delay. (g) To train teachers become qualified seed teachers in the itinerant guidance. (h) To assist teachers screening and reporting of suspected individual cases.

The implementation plan for nursery institutions to accept children with developmental delay: When common kindergartens or nurseries accept children with developmental delay or with mentally or physically disability, most of them have to purchase relevant teaching materials, or even have to improve their hardware facilities to meet the special needs of these children on their development states and education. In order to encourage kindergartens and nurseries to admit special children, the government actively assists these nursing institutions in building appropriate facilities and environments for inclusive education or nursing services, and enhancing their willingness and ability to grant admittances to preschool children with developmental delay (including those with certificates of mentally or physically disabled), subsidizing them on facilities and equipments for children with developmental delay such as sensory integration instruments, teaching materials and teaching aids, cameras \& video recorders, computers and software, communication aids, equipments used in psychological tests and psychological counseling, nursing and recreational facilities, barrier-free facilities, etc.

The Head Start from foreign spouse families or disadvantaged families: This plan is to assist cultural disadvantaged children and children from foreign spouse families or disadvantaged families on their linguistic development with necessary linguistic education. Through tutorial practices at home, environmental design, reading guidance, demonstration of language expression, or by means of parent-child reading based on groups and growth group, etc, trainers can strengthen the early linguistic development of the child and help the guardians to understand the linguistic development of the child, provide an advantageous environment for the cognitive development of the child and thus to further narrow the gap between him/her and ordinary children.

The Implementation plan for the integration of early intervention resources for children with developmental delay: According to the convenience and accessibility of the local early intervention resources, each local government will integrate and exchange achievement in hygiene, education and social administration resources under its jurisdiction, plan for division of labor, improve the regional service function of the EIS by means of regional joint report, the sharing of management methods of individual cases, coproduction of propaganda leaflets, resources manuals, cooperative handling of screening activities, publicizing, researches and trainings, etc.

Individual cases management system of early intervention service for children with developmental delay: The CWB has completed the development of an individual cases management system of EIS for children with developmental delay. By establishing a tracing and guidance mechanism to collect information from 25 report \& referral centers of local governments, the management system can have the dynamic states of each individual case well in hand and provide complete EIS resources.

The holding of professional training courses and propaganda activities: The CWB will plan and produce unified propaganda leaflets, video tapes, VCDs, video clips to strengthen propaganda of the notion and the practices of the discovery and intervention of these children at the earliest possible time via media, and will guide local governments and civil organizations to open training courses for relevant workers and to hold propaganda and screen activities through children's 
games.

The establishment of services transition and cooperation mechanisms: Through the opening of periodical promotion conferences for early intervention and the integration of early intervention resources of medical, educational departments and local governments, the EIS can become even more complete. By relevant researches, we can dig out the needs of children with developmental delay and provide appropriate handling models and parent education courses, and a more perfect EIS system is expected.

\section{Statistics of the Achievements of Early Intervention in 2000-2006}

The EIS for children with developmental delay in Taiwan is growing steadily. Let's take the report rate of children with developmental delay as an example: this report rate grows from the $3.12 \%$ of 2000 to $5.63 \%$ of 2006, which indicates that through relevant publicity and education, the public gradually attaches importance to the development of children; the number of individual cases under management grows from 6,552 to 15,432 with a 2.3 times increase approximately. Through the increasing of intervention bases and the subsidies to expenses on intervention and transportation, children receive intervention services increase from 7,436 to 20,743 , leading to approximately 2.78 times in growth.

\section{Review and Perspective}

The core of the spirit for early intervention is that by early screening, early discovery and appropriate early intervention measures, the seriousness of the developmental delay and the disability of the child can be alleviated, and the burden and pressure to the family during the process of the intervention of the child can be lightened. In comparison to the development of other welfare items, the history of the promotion of EIS in children with developmental delay is not too long. However, under the cooperation of the government and civil organizations, we have come a long way in this field. Looking into the future, we will continue to make more efforts in the following points:

\section{To Strengthen the Publicity and Education Work so as to Attract the Attention of the Public}

The government has done quite a lot on the publicizing of the importance of and has provided relevant supportive measures to the development of

\section{Table 2}

Annual Statistics of Children with Developmental Delay Receiving Early Intervention Unit: head

\begin{tabular}{|c|c|c|c|c|}
\hline Year & Report number & Report percentage (\%) & $\begin{array}{l}\text { Number of case } \\
\text { management }\end{array}$ & $\begin{array}{c}\text { Number of children receiving } \\
\text { early intervention }\end{array}$ \\
\hline 2000 & 9,421 & 3.12 & 6,552 & 7,436 \\
\hline 2001 & 11,981 & 4.11 & 7,698 & 9,350 \\
\hline 2002 & 13,264 & 4.75 & 11,609 & 16,286 \\
\hline 2003 & 13,231 & 5.03 & 12,442 & 17,909 \\
\hline 2004 & 11,778 & 4.63 & 13,327 & 20,318 \\
\hline 2005 & 12,176 & 5.03 & 14,642 & 17,997 \\
\hline 2006 & 12,688 & 5.63 & 15,432 & 20,743 \\
\hline 2007.06 & 6,815 & 3.10 & 14,059 & 15,253 \\
\hline
\end{tabular}

Note. From Child welfare data [public notice] Child Welfare Bureau, 2006 
children in recent years in the hope that children with abnormality can be discovered at the earliest possible time; however, some parents still hold the traditional opinion that "greater talent shows itself late in life.," or they might be reluctant to face the reality that their children are developmentally delayed. As a result, the opportunity to receive early intervention is delayed. Therefore, we should increase relative publicity work on the importance of early intervention in children with developmental delay and hygiene education among pregnant women and parents of new-born babies so as to make the parents become more sensitive to the development of the children. Furthermore, the government has set up the month of April as month of screening for children with developmental delay and has unified and strengthened its propaganda to draw the public's attention. And this will be helpful to the early detection of children with abnormalities.

\section{To Discover Children with Abnormality in Development at the Earliest Possible Time and Lower the Age of Reporting}

The most important point of early intervention is the intervention at the earliest possible time before the children attain the age of 3 when their nervous systems and other physiological systems still not develop into mature stage. Therefore, we will implement the preventive healthcare service for children in the future and achieve the early discovery and timely report of children with abnormalities, so as to facilitate the timely intervention services of professionals. Especially for children between 0-2 who are still cared by their parents or babysitters, through the "community babysitter system" which is comprised of babysitters with professional certificates, by strengthen on-the-job education to babysitters on the early intervention of children with developmental delay, with the cooperation of the promotion of "the manual of children's health" into kindergartens and nurseries, teachers and nursing workers of kindergartens and nurseries can use the simple measurement scale for the screening of children with developmental delay to carry out initial screening on children, so that suspected cases of children with developmental delay can be reported at the earliest possible time, and the early detection and early intervention can be fulfilled.

\section{To Strengthen the Manpower of the Professional Team and to Exert More Efforts on the Intervention Service, so as to Improve the Quality of the Service}

Children receive early intervention can not achieve desired effects in just one move, and whether this service is conveniently accessible can even affect the parents' willingness to take part in early intervention. Presently EIS can be available from medical sites or early intervention organizations, etc. However, EIS are still very scarce in some outlying islands, remote areas and in some indigenous tribes. Despite that there are at-home services available that can make up for this deficiency, long-term plans for the training of early intervention professionals such as speech therapists, clinical psychologists should still be implemented. Furthermore, the assurance and improvement of service quality will be the future direction of the development of EIS for children with developmental delay.

\section{To Strengthen the Connections among Different Systems and Departments and to Provide All-Round Care and Transition}

A transition represents a process of change in life or study environment, such as the transition processes of the providers and service personnel of EIS. For example, when a child is admitted to a kindergarten, or when a child is admitted to a primary school, he/she can choose the transition service to keep the continuity of his/her EIS. The transition service models for children with developmental delay and their families should be established on the basis of the researches and the reviews of practical work by professional teams on the three systems of social welfare, healthcare and education. 


\section{Conclusion}

EIS can improve delay in development of children. It can also give the family more support, so that the family would function better. In order to reduce the children's dependence on the society and the possibilities of being sent to an asylum, the promotion of early intervention is necessary. If the early intervention is successful, the children might no longer be in urgent need for a special education class or special education service after they attend a school. This can also reduce medical expenses and education costs for the family (Wanne, 2001; Lin, 1998). The screening and early intervention of children with developmental delay need the integration of various relevant specialties and the cooperation of several teams from different fields (including education, healthcare, social administration, hospitals, organizations, kindergartens and nurseries) and government support. As regards to the establishment and improvement of the report and monitoring system for children with developmental delay, these also depend on the attention from the government and the vigorous promotion of the government. To the parents of these children with developmental delay, in the process of the growth, problems that can not be solved by people in any single professional field will come in succession. Therefore, when it comes to the EIS for the children with developmental delay, we must break the barriers between everyone, and carry out inter-discipline cooperation and take the needs of the children and their family as the central focus, so as to provide the most appropriate service to the children. Every child has only one childhood; no one can grow and develop all over again from the start. Therefore, early intervention should start as early as possible. Early intervention can stir the latent energy of the individual life. The promotion of early intervention is a very important topic which needs related departments' hard work. The government in Taiwan will keep struggling on the current basis, actively coordinate the cooperation of the departments to plan and promote the business of EIS, so as to benefit children with developmental delay and bring the child welfare of our nation to a further milestone.

\section{References}

Child and Youth Welfare Law (2003 Amendment).

Child Welfare Bureau, Ministry of the Interior. (2006). Implementation plan for the itinerant guidance for nursery institutions to accept children with developmental delay [Public Notice]. Taichung City: Child Welfare Bureau, Ministry of the Interior. September 10th, the 96th year of ROC. Retrieved from http://www.cbi.gov.tw/file downloading area.

Child Welfare Bureau, Ministry of the Interior. (2006). Service plan of the formative education for preschool children from foreign spouse families or disadvantaged families [Public Notice], Taichung City: Child Welfare Bureau, Ministry of the Interior. September 10th, the 96th year of ROC. Retrieved from http://www.cbi.gov.tw/file downloading area.

Child Welfare Bureau. (2006). Ministry of the Interior Child Welfare Data [Public Notice]. Taichung City: Child Welfare Bureau, Ministry of the Interior. September 10th, the 96th year of ROC. Retrieved from http://www.cbi.gov.tw/ Child Welfare Data / Early Intervention.

Child Welfare Bureau, Ministry of the Interior. (2006). Implementation plan of the home-service for children with developmental delay [Public Notice], Taichung City: Child Welfare Bureau, Ministry of the Interior. September 10th, the 96th year of ROC. Retrieved from http://www.cbi.gov.tw/file downloading area.

Child Welfare Bureau, Ministry of the Interior. (2006). Implementation plan of the early intervention service for children with developmental delay [Public Notice], Taichung City: Child Welfare Bureau, Ministry of the Interior. September 10th, the 96th year of ROC. Retrieved from http:// www.cbi.gov.tw/ file downloading area.

Child Welfare Bureau, Ministry of the Interior. (2006). Implementation plan for nursery institutions to 
accept children with developmental delay [Public Notice], Taichung City: Child Welfare Bureau, Ministry of the Interior. September 10th, the 96th year of ROC. Retrieved from http://www. cbi.gov.tw/file downloading area.

Child Welfare Law (1993 Amendment).

Guo, H. (1996). Early intervention for children with developmental delay. Acta Paediatrica Sinica R.O.C, 37(A), 19-27.

Lin, H. (2001). Interview reports of the report \& referral centers cum Team Evaluation centers for children with developmental delay in Taiwan-Fuchien region. The meeting practice of the report \& referral centers cum Team Evaluation centers for children with developmental delay in TaiwanFuchien region 1-17, Taipei: Parents' association for persons with intellectual disability, R.O.C.

Lin, L. (1998). Developmental delay needs treatment at the earliest possible time. Social Welfare, 134, 6264.

Special Education Law (1997 Amendment).

Wang, G. (1996). The analysis of the laws of Taiwan and relevant theoretical models of the early intervention service policy for mentally or physically disabled children Soochow. Journal of Social Work, 2, 333-350.

Wanne, Y. W. (2001). The study of early intervention referral center for the developmental children (NOI-VBI-089-002). Taichung City: Child Welfare Bureau, Ministry of the Interior.

Yi, M. (2004). Early intervention and children with developmental delay from Hand in hand, we are all good friends-preschool inclusion education practice workbook. Taipei City: The first social welfare foundation, a non-profitable organization.

Zhang, X. (2002). Exploration on factors that affect the execution of report and referral policies of early intervention services. Community Development Journal, 97, 329-341. 